


\title{
Bitcoin economic behavior analysis and policy implications by leveraging deep learning and high-frequency data
}

\author{
Vasilis Siakoulis ${ }^{1}$, Anastasios Petropoulos ${ }^{2}$ and Panagiotis Lazaris ${ }^{3}$ \\ ${ }^{1}$ Bank of Greece, 3 Amerikis, 10250 Athens, Greece, E-mail: vsiakoulis@bankofgreece.gr \\ ${ }^{2}$ Bank of Greece, 3 Amerikis, 10250 Athens, Greece, E-mail: apetropoulos@bankofgreece.gr \\ ${ }^{3}$ Bank of Greece, 3 Amerikis, 10250 Athens, Greece, E-mail: plazaris@bankofgreece.gr
}

\section{Article Info}

Volume 1, Issue 1, February 2021

Received : 22 November 2020

Accepted : 03 January 2021

Published : 05 February 2021

doi: 10.51483/IJDSBDA.1.1.2021.55-62

\begin{abstract}
The recent surge in Bitcoin price performance has attracted significant attention from both the market and academic researchers. This paper constitutes the first principled attempt to determine market risk own-funds requirements for Bitcoin. To this end, we examine price microstructure of the USD per bitcoin, and compare to other financial variables, as a proxy toward classifying Bitcoin into the appropriate risk-class. Using the outcomes of this analysis, we classify and quantify the entailed risk from a market risk minimum capital requirements perspective. To perform the prescribed analysis, we introduce a novel methodological paradigm, which adopts bleeding-edge concepts from the field of Data Science and Machine Learning.

Keywords: Bitcoin, Cryptocurrencies, Extreme gradient boosting, Deep neural networks

(C) 2021 International Journal of Data Science and Big Data Analytics. This is an open access article under the CC BY license (https://creativecommons.org/licenses/by/4.0/), which permits unrestricted use, distribution, and reproduction in any medium, provided you give appropriate credit to the original author(s) and the source, provide a link to the Creative Commons license, and indicate if changes were made.
\end{abstract}

\section{Introduction}

Bitcoin was invented by an unknown person or group of people using the name Satoshi Nakamoto and released as open-source software in 2009. It is the first form of decentralized digital currency, typically referred to as cryptocurrencies. It is decentralized in the sense that the system works without the intervention of a central bank or a supervisory authority. It works as a peer-to-peer network, under which transactions take place directly between users, without intermediaries. Transaction verification is performed on network nodes by means of cryptographic protocols. All transactions are recorded in a public distributed ledger called the blockchain. Bitcoins are created as a reward for a process known as mining. They can be exchanged for other currencies, products, or services. An estimate provided by the University of Cambridge reckons that in 2017, there were 2.9 to 5.8 million unique users using a cryptocurrency wallet, with the vast majority using Bitcoin.

The recent considerable increase in Bitcoin price performance has attracted significant attention from both the market and academic researchers. Indeed, Bitcoin currently accounts for over $40 \%$ of the total value of cryptocurrency

\footnotetext{
* Corresponding author: Vasilis Siakoulis, Bank of Greece, 3 Amerikis, 10250 Athens, Greece. E-mail: vsiakoulis@bankofgreece.gr
} 
transactions. On March 2, 2018, the Governor of the Bank of England, and Chair of the Financial Stability Board, Mark Carney, gave a conference speech at Edinburgh University. During it, he stated that "the time has come to hold the crypto-asset ecosystem to the same standards as the rest of the financial system." However, little is currently known regarding the properties of cryptocurrency assets. Recent literature has examined the validity of the martingale hypothesis, as well as the hypothesis of market efficiency when it comes to Bitcoin price behavior (Bariviera, 2017; Chea et al., 2018; Urquhart, 2017). According to these studies, the Bitcoin market remains inefficient. Other preliminary results on Bitcoin support the long memory hypotheses regarding its volatility structure (Katsiampa, 2017; Lahmiri et al., 2018). Such a behavior indicates a significant speculative component that typically leads to bubble behavior, increased leverage effects (Chea et al., 2018; MacDonnel, 2014; Phillip et al., 2018; Urquhart, 2016), and pricing inconsistencies (Pieters et al., 2017), in cases of failure to establish an effective regulation framework. Finally, other recent studies have shown that return forecasting is possible due to market inefficiency (Balcilar et al., 2017; Demir et al., 2018; Katsiampa, 2017); this essentially implies that Bitcoin offers significant arbitrage opportunities, as it gives rise to predictable patterns that can be easily captured in an algorithmic fashion.

In May 2012, the Basel Committee published the first consultative document regarding the Fundamental Review of the Trading Book (FRTB) capital requirements. Several consultative papers were published by the Committee which concluded to the publication of new standards in January 2016. One of the main amendments performed over the previous standards was the incorporation of different liquidity horizons across asset markets; these replaced the static 10-day horizon. Under the new internal model approach, and in order to capture diverse market liquidity levels of various assets, it is stipulated that all risk factors be mapped to the appropriate liquidity horizon; this may range from 10 to 120 days.

Despite these advances, the regulatory framework does not specifically account for the risk attached to Bitcoin or other cryptocurrencies. (EBA note on cryptocurrencies and BIS). Specifically, both the current framework on own-funds requirements for market risk, as well as its proposed amendments, fail to provide specific guidelines regarding the treatment of positions in virtual currencies. The aim of this work is to address this profound lack, by determining market risk own-funds requirements. To this end, we examine price microstructure of the USD per Bitcoin, and compare to other financial variables, as a proxy toward classifying Bitcoin into the appropriate risk-class. In tandem, we aspire to obtain accurate estimates of the appropriate liquidity horizon for Bitcoin (if any); to this end, we utilize the liquidity horizons included in the table of paragraph $181(\mathrm{k})$ of the market risk standards published by BIS.

From the methodological point of view, the novelty of our devised framework consists in leveraging bleeding-edge technologies from the field of Data Science and Machine Learning. Specifically, we use deep learning methods, appropriately combined with extreme boosting algorithms in a novel way, applied to high-frequency data of Bitcoin prices. This unique combination of technologies allows for effectively understanding the dynamic interdependences of Bitcoin prices and comparing them to the properties of other financial assets. Indeed, high-frequency data allow for an in-depth investigation of the microstructure of the Bitcoin market, as well as for addressing intraday patterns, effects in time-varying volatility, and temporal dependencies in return time series. At the same time, the proposed combination of bleeding-edge Machine Learning techniques is expected to allow for disentangling microstructure noise from highfrequency observations of asset prices; this way, we anticipate our methods will be able to detect forecasting patterns that enable reliable testing of the efficient market hypothesis.

Our adopted methodology relies on the analysis of a large dataset of high frequency data. Our analysis targets market liquidity and volatility patterns, with the aim of classifying and quantifying the entailed risk from a market risk minimum capital requirements perspective. Specifically, to address the risk underlying Bitcoin positions, we attempt to model the temporal properties of high frequency data, and compare them against other groups of financial assets, namely currencies, commodities, and stocks.

To this end, we leverage bleeding-edge deep learning algorithms, Dropout networks (Kingma et al., 2015) with Rectified Linear Units (ReLU), (Vinod and Hinton, 2010), implemented in the MXNET package. Dropout ReLU networks are a key deep learning method used in modeling and learning to predict temporal dynamics in time-series data of arbitrarily high dimensionality. They have been extremely successful in as diverse time-series modeling tasks as machine translation (Cho et al., 2014; Tu et al., 2016), machine summarization (See et al., 2017), video understanding (Yao et al., 2015), and recommendation engines (Quadrana et al., 2017). As such, they offer an extremely potent means of discovering patterns appropriate for predicting Bitcoin price. According to the efficient market hypothesis, there should be no such patterns. Under this hypothesis, a GRU trained on data comprising a significant number of lags should not achieve forecasting performance above an area under ROC (AUROC) measure of 50\%. The AUROC of a classifier is equivalent to the probability that the classifier will rank a randomly chosen positive instance higher than a randomly chosen 
negative instance. In practice, the value of AUROC varies between 0.5 and 1 , with a value above 0.8 denoting a very good performance of the algorithm. Hence, an AUROC value close to 50\% essentially indicates an algorithm incapable of generating anything better than random decisions. The opposite occasion, whereby the trained GRU may achieve predictive performance well above $50 \%$, would, therefore, be a strong indicator of the existence of strong temporal patterns, which invalidate the efficient market hypothesis.

On top of these developments, our methodology is additionally augmented with the development of algorithms that model microstructure series. Specifically, we train eXtreme Gradient Boosting (XGBOOST) algorithms, as these are widely used for liquid and less liquid currencies, equities and commodities. Then, we examine the success rate of the trained model of each asset against Bitcoin forecasting accuracy.

The outcomes of our experimental analysis provide a unique deep understanding of the market risk components in Bitcoin market; these outcomes can be useful for informing future regulatory initiatives. Indeed, there is currently an urgent need of preventing inflated Bitcoin prices and market inefficiency, as they induce immense risks to investors and potentially to the financial stability. Thus, our work contributes to this increasing need for enriching the current regulatory framework; it addresses the regulatory treatment of cryptocurrencies by financial institutions and offers a guide for quantifying their risk under a capital adequacy framework.

\section{Literature review}

There have been several attempts to model the behavior of Bitcoin by analyzing daily data of prices as well as connections with other financial variables. To this end, researchers typically employ techniques also applied to traditional financial markets and variables. Such an approach is also reasonable in the sense that it allows for extracting similarities among Bitcoin and other financial markets characteristics.

In one of the earliest such works, MacDonell (2014) tried to identify the factors that drive the value of Bitcoin prices. Concurrently, this work also tested if it could be possible to predict impending crashes ex-ante. It was found that Bitcoin prices are inversely related with VIX values; this essentially suggests that investors behavior is irrelevant to Bitcoin price fluctuation. Thus, the aforementioned inverse relationship is a strong indicator of the fact that investors choose to invest money in Bitcoin when looking for higher returns, as opposed to seeking a shelter for their wealth. Finally, the application of Log Periodic Power Law models in the same work showed that this simple statistical modeling approach is capable of predicting the crash in Bitcoin prices that occurred in December 2013.

On a different vein, Cheah and John Fry (2015) used mathematical techniques originated in physics in an attempt to answer whether the boom-bust cycle of Bitcoin prices is a bubble, as well as whether its fundamental value is non-zero. They concluded that, similar to other asset classes, Bitcoin prices are exposed to speculators, and that its prices already carry the bubble component. Last but not least, they also found that the fundamental value of Bitcoin is, indeed, zero.

Another significant subject that is worth of investigation concerns whether Bitcoin exhibits price clustering tendencies. Price clustering is a behavioral aspect that is well-known to characterize many financial markets, including spot foreign exchange market, commodity markets, and stock markets. Therefore, since little is known about Bitcoin prices, Urquhart (2017) examined Bitcoin prices clustering. He found significant evidences of price clustering around whole numbers, with over $10 \%$ of prices ending with decimal digits of 00 , as well as the correlation patterns between Bitcoin price clustering and its price and volume.

Further, Bariviera (2017) used daily price data of Bitcoin from 2011 to 2017 to estimate the Hurst exponent. To this end, they employed both R/S and Detrended Fluctuation Analysis (DFA) methods on the daily logarithmic return and the daily price volatility. Based on the outcomes of these analyses, and considering DFA to be more appropriate, it was observed that daily returns suffered a regime switch in 2014; the new regime corresponded to a more efficient market. At the same time, daily volatility was shown to exhibit a steady long-range memory.

Similar findings on the long memory properties of cryptocurrencies were shown in Phillip et al. (2017). In addition, the leverage effect that was firstly introduced by F. Black in 1976 was shown to also be present in cryptocurrency prices. Specifically, they used generalized autoregressive conditional heteroscedastic models to measure time-varying volatility in prices of Bitcoin; on these grounds, volatility estimation showed the presence of a stochastic volatility process. Lastly, similar to other financial series, their distributions were found to be heavy tailed. Along the same lines, Cheah $e t$ al. (2018) also pinpointed this long memory feature, by performing analysis of daily Bitcoin price data. They stressed that this property could cause a "system failure" in the event of the application of new regulation in financial markets. Moreover, the showed that the Bitcoin market is inefficient, which, in conjunction with the long memory characteristic of the prices, could be exploited by speculators to gain profits.

Finally, Balcilar et al. (2017) examined the relationship of Bitcoin returns and volatility with its traded volume, similar 
to analyses performed for other financial markets (e.g., stocks, bonds, commodities interest rates, etc). To this end, they used daily data covering the period of December 2011 to April 2016 exchanged on Bitstamp. They showed that, as long as the Bitcoin market is under the normal mode, volume can predict returns; yet, when the Bitcoin market is out/over performing, only the past values can matter for predicting future returns. Regardless of the market mode, the volume exchanged does not predict the Bitcoin price volatility.

\section{Data collection and methodology}

We employ minute data on Bitcoin price, spanning the period 8/5/2017 - 3/3/2018 (the period is selected with the purpose of reducing market correlation effects); these are obtained from DUKASCOPY website 4 . Due to our limited access to minute data pertaining to other assets, we compare Bitcoin spot prices behavior to a set of 25 risk factors pertaining to equity large cap, precious metals and non-ferrous metals prices, FX liquid currency pairs, and less liquid currency pairs; the considered asset types by category are detailed in Table 1 . The used data points pertaining to the considered factors (assets) span the same period as the used Bitcoin price data.

\begin{tabular}{|c|c|c|}
\hline Sign & Description & Class \\
\hline GAS & Natural Gas & Commodity \\
\hline XAG & Spot Silver & Commodity \\
\hline $\mathbf{X A U}$ & Spot Gold & Commodity \\
\hline BRENT & US Brent Crude Oil & Commodity \\
\hline COPPER & High Grade Copper & Commodity \\
\hline GBP & British Pound & Liquid Currency \\
\hline JPY & Japan Yen & Liquid Currency \\
\hline MXN & Mexican Peso & Liquid Currency \\
\hline NZD & New Zealand Dollar & Liquid Currency \\
\hline ZAR & South African Rand & Liquid Currency \\
\hline AUD & Australian Dollar & Liquid Currency \\
\hline CAD & Canadian Dollar & Liquid Currency \\
\hline CHF & Swiss Franc & Liquid Currency \\
\hline EUR & Euro & Liquid Currency \\
\hline ILS & Israel Sekel & Less Liquid Currency \\
\hline THB & Thai Baht & Less Liquid Currency \\
\hline PLZ & Polish Zloty & Less Liquid Currency \\
\hline CZK & Czech Koruna & Less Liquid Currency \\
\hline HUF & Hungarian Fiorine & Less Liquid Currency \\
\hline GB R & UK 100 Index & Stock \\
\hline JPN & Japan 225 & Stock \\
\hline USA500 & USA 500 Index & Stock \\
\hline DEU & Germany 30 Index & Stock \\
\hline EDP & Energia de Portugal SA & Stock \\
\hline GALP & Galp Energia SGPS & Stock \\
\hline
\end{tabular}

4 https://www.dukascopy.com/swiss/english/marketwatch/historical/ 
In order to study volatility patterns, we employ the squared return as a historical volatility proxy. Under this modeling framework, large positive and negative minute movements yield high squared returns. We also employ the bidask spread to approach the liquidity depth of each asset. We note that the size of the bid-ask spread from one asset to another differs mainly because of the difference in the liquidity of each asset. Certain markets are more liquid than others. For example, currency is considered the most liquid asset in the world, and the bid-ask spread in the currency market is one of the smallest (one-hundredth of a percent); in other words, the spread can be measured in fractions of pennies. On the other hand, less liquid assets, such as small-cap stocks or less liquid currencies, may have spreads that are equivalent to 1 to $2 \%$ of the assets lowest ask price. For instance, Mosbeh and Nidhal (2016) attempted to link liquidity with the market structure of the Tunis stock market using data over the 2005 to 2011 time period. As expected, the results showed that liquidity level is inversely connected to bid-ask spread levels.

Our volatility modeling approach is the following: For each of the aforementioned assets (Table 1), an XGBOOST model (Chen et al., 2016) is trained on both volatility and bid ask spread data. Our used training sample constitutes a 75\% subsample of the available dataset. On the other hand, the remained $25 \%$ of the available data is used for testing the discriminatory power of the model (test sample). This is affected through the AUROC metric on the test sample. In all cases, the used independent variables (covariates) comprise the last fifty lags; in order to avoid convergence issues, the dependent variables are discretized to binary, i.e., $\{0,1\}$, based on the subsample median.

Having trained the models pertaining to the considered assets, we then proceed to examine Bitcoin behavior. Our analysis consists in using the models trained on the previous assets to perform prediction in the available Bitcoin dataset; performance is measured on the grounds of the AUROC metric. It is expected that the models pertaining to asset classes with similar microstructure behavior with Bitcoin will exhibit higher AUROC metric in the Bitcoin test sample. This will allow for us to make a comparative assessment on the appropriate Bitcoin classification into one of the commodity, currency, less liquid currency, or stock classes.

Finally, in order to delve deeper into price discovery behavior, we examine the market efficiency hypothesis of the Bitcoin through the use of an appropriate popular form of deep learning models, specifically, Dropout networks with ReLU nonlinearities. According to the efficient market hypothesis, there should be no patterns which could be exploited by a deep learning model to predict the Bitcoin price with statistically significant accuracy. We use the same break proportion ( $75 \%$ development sample $-25 \%$ testing sample) so as to train the aforementioned model and test the market efficiency hypothesis.

\section{Model development}

In the last few years, machine learning has experienced a wave of record-breaking innovations through the development of a family of methods inspired by the hierarchical operation of the cortex, namely deep learning approaches. Deep learning networks have dramatically improved the state-of-the-art in as diverse tasks as speech recognition, visual object recognition, drug discovery and genomics. Deep neural networks (DNNs) constitute large-scale neural networks that employ appropriate nonlinear activation functions. Indeed, the employed nonlinear activation functions constitute an essential component of DNNs.

Constructing a DNN without nonlinear activation functions is impossible, as without these the deep architecture collapses to an equivalent shallow one. Typical choices are logistic sigmoid, hyperbolic tangent and rectified linear unit (ReLU). The logistic sigmoid and hyperbolic tangent activation functions are closely related; both belong to the sigmoid family. A disadvantage of the sigmoid activation function is that it must be kept small due to their tendency to saturate with large positive or negative values. To alleviate this problem, practitioners have derived piecewise linear units like the popular ReLU, which are now the standard choice in deep learning research.

On a different perspective, since DNNs comprise a huge number of trainable parameters, it is key that appropriate techniques be employed to prevent them from overfitting. Indeed, it is now widely understood that one of the main reasons behind the explosive success and popularity of DNNs consists in the availability of simple, effective, and efficient regularization techniques, developed in the last few years. Dropout has been the first, and, expectably enough, the most popular regularization technique for DNNs. In essence, it consists in randomly dropping different units of the network on each iteration of the training algorithm. This way, only the parameters related to a subset of the network units are trained on each iteration; this ameliorates the associated network overfitting tendency, and it does so in a way that ensures that all network parameters are effectively trained. 
Inspired from these merits, we employ Dropout DNNs with ReLU activations to train and deploy DNNs, with the purpose of testing the market efficiency hypothesis. Specifically, we use the Apache MXNET toolbox of R, available in https://mxnet.incubator.apache.org/api/r/index.html. Our paper constitutes the first work presented in the related literature on Bitcoin nature that considers application of deep learning to address this challenging financial modeling task. We postulated deep networks that are up to five hidden layers deep and comprise various numbers of neurons. Model selection using cross-validation was performed by maximizing the AUROC metric.

On the other hand, given the extensive number of employed predictors (50 lags) and the large-scale dataset employed (minute data) we resort XGBOOST for comparing the market liquidity and volatility patterns of Bitcoin with other assets. The advantage of Boosting consists in the fact that it reduces predictive variance, as well as predictive bias. Specifically, variance is reduced by making use of multiple models (bagging), such as Random Forests. On the other hand, bias is reduced by training the set of postulated models in a sequential fashion, whereby the next trained model is presented with the error signals of the last trained one (boosting). Specifically, under the gradient boosting rationale, each subsequent model is trained using the residuals of previous models, that is the difference between the predicted and true values.

XGBoost is an advanced implementation of gradient boosting, offering increased efficiency, accuracy and scalability compared. It supports fitting various kinds of objective functions, including regression, classification and ranking. XGBoost offers increased flexibility, since optimization is performed on an extended set of hyperparameters, while it fully supports online training, without the danger of catastrophic forgetting. For the purposes of our work, we implemented XGBoost by utilizing the XGBoost R package. We performed an extensive cross-validation procedure to select a series of entailed hyper parameters, including the maximum depth of trees generated, the minimum leaf nodes size to perform a split, and the size of sub-sampling for building the classification trees and the variables considered in each split. The objective function used for the current problem was binary cross-entropy, due to the binary nature of the dependent variable. We used AUROC metric for model selection in the context of cross-validation.

From the results (Table 2) we see that the trained XGBoost models exhibit good discriminatory power on out-ofsample basis. Specifically, out-of-sample AUROC metrics surpass $68 \%$ accuracy in terms of volatility prediction, and $77 \%$ accuracy when it comes to market liquidity. Concerning liquidity depth, our results show that Bitcoin exhibits the highest average AUROC when prediction is performed using the XGBOOST models trained on less liquid currencies. The second-best performer are models trained on liquid currencies and commodities, while stock-driven models are the worst performers on average. Turning to volatility prediction, no material differences are observed among XGBOOST models trained on different types of assets. This is strong indication that a VaR approach may be as effective for the purpose of Bitcoin risk monitoring as it is in the context of other asset types. Finally, regarding our examination of the market efficiency hypothesis, it turns out that the trained Dropout ReLU network (Apache MXNET) yields an out-of sample AUROC metric of 55.21\% (in sample fit 56.88\%). This proves that Bitcoin price fluctuation entails strong patterns that can be exploited for the purpose of price discovery. This finding leads to the unequivocal conclusion that the Bitcoin market is not an efficient one.

Table 2: AUROC measures for Volatility (Vol) and Spread for each asset class. In-sample (training sample for XGBOOST development); out-of-sample (test sample for XGBOOST evaluation). Vol-Bitcoin and Spread - Bitcoin are the respective AUROC measures when the Bitcoin minute data are employed as test sample in each asset classes trained XGBOOST model.

\begin{tabular}{|c|c|c|c|c|c|c|c|c|}
\hline Sign & Description & Class & $\begin{array}{c}\text { Vol-In } \\
\text { sample }\end{array}$ & $\begin{array}{l}\text { Vol-Out } \\
\text { sample }\end{array}$ & $\begin{array}{c}\text { Vol- } \\
\text { Bitcoin }\end{array}$ & $\begin{array}{c}\text { Spread- } \\
\text { In sample }\end{array}$ & $\begin{array}{c}\text { Spread-Out } \\
\text { sample }\end{array}$ & $\begin{array}{l}\text { Spread- } \\
\text { Bitcoin }\end{array}$ \\
\hline GAS & Natural Gas & Commodity & $67.8 \%$ & $63.6 \%$ & $88.4 \%$ & $84.4 \%$ & $83.8 \%$ & $73.8 \%$ \\
\hline GBP & British Pound & Liquid Currency & $72.9 \%$ & $71.1 \%$ & $86.8 \%$ & $86.7 \%$ & $85.9 \%$ & $62.5 \%$ \\
\hline GB R & UK 100 Index & Stock & $67.9 \%$ & $66.7 \%$ & $86.1 \%$ & $97.9 \%$ & $96.7 \%$ & $74.6 \%$ \\
\hline ILS & Israel Sekel & Less Liq. Currency & $73.5 \%$ & $70.5 \%$ & $88.3 \%$ & $94.4 \%$ & $94.1 \%$ & $93.0 \%$ \\
\hline JPN & Japan 225 & Stock & $64.9 \%$ & $63.7 \%$ & $88.2 \%$ & $97.9 \%$ & $95.0 \%$ & $79.8 \%$ \\
\hline JPY & Japan Yen & Liquid Currency & $69.9 \%$ & $68.0 \%$ & $85.6 \%$ & $83.2 \%$ & $82.4 \%$ & $76.4 \%$ \\
\hline MXN & Mexican Peso & Liquid Currency & $75.0 \%$ & $73.2 \%$ & $87.4 \%$ & $96.8 \%$ & $96.8 \%$ & $81.7 \%$ \\
\hline
\end{tabular}




\begin{tabular}{|c|c|c|c|c|c|c|c|c|}
\hline Sign & Description & Class & $\begin{array}{c}\text { Vol-In } \\
\text { sample }\end{array}$ & $\begin{array}{l}\text { Vol-Out } \\
\text { sample }\end{array}$ & $\begin{array}{c}\text { Vol- } \\
\text { Bitcoin }\end{array}$ & $\begin{array}{c}\text { Spread- } \\
\text { In sample }\end{array}$ & $\begin{array}{c}\text { Spread-Out } \\
\text { sample }\end{array}$ & $\begin{array}{l}\text { Spread- } \\
\text { Bitcoin }\end{array}$ \\
\hline NZD & New Zealand Dollar & Liquid Currency & $67.4 \%$ & $65.4 \%$ & $86.6 \%$ & $85.3 \%$ & $84.4 \%$ & $73.7 \%$ \\
\hline THB & Thai Baht & Less Liq. Currency & $73.4 \%$ & $70.6 \%$ & $87.2 \%$ & $90.0 \%$ & $77.9 \%$ & $91.2 \%$ \\
\hline USA500 & USA 500 Index & Stock & $70.1 \%$ & $66.5 \%$ & $87.0 \%$ & $98.9 \%$ & $97.4 \%$ & $53.1 \%$ \\
\hline XAG & Spot silver & Commodity & $65.7 \%$ & $62.3 \%$ & $87.3 \%$ & $94.3 \%$ & $93.8 \%$ & $70.5 \%$ \\
\hline $\mathbf{X A U}$ & Spot gold & Commodity & $66.7 \%$ & $65.9 \%$ & $87.6 \%$ & $84.3 \%$ & $84.2 \%$ & $72.9 \%$ \\
\hline ZAR & South African Rand & Liquid Currency & $77.4 \%$ & $76.1 \%$ & $87.7 \%$ & $97.4 \%$ & $97.4 \%$ & $91.1 \%$ \\
\hline AUD & Australian Dollar & Liquid Currency & $67.9 \%$ & $65.3 \%$ & $84.1 \%$ & $81.2 \%$ & $80.2 \%$ & $71.4 \%$ \\
\hline BRENT & US Brent Crude Oil & Commodity & $72.5 \%$ & $70.3 \%$ & $88.1 \%$ & $97.8 \%$ & $97.3 \%$ & $69.9 \%$ \\
\hline CAD & Canadian Dollar & Liquid Currency & $71.1 \%$ & $69.2 \%$ & $85.3 \%$ & $84.7 \%$ & $83.6 \%$ & $69.4 \%$ \\
\hline CHF & Swiss Franc & Liquid Currency & $70.8 \%$ & $70.1 \%$ & $87.9 \%$ & $89.9 \%$ & $89.0 \%$ & $66.9 \%$ \\
\hline COPPER & High Grade Copper & Commodity & $69.7 \%$ & $66.5 \%$ & $85.8 \%$ & $94.4 \%$ & $93.9 \%$ & $72.6 \%$ \\
\hline DEU & Germany 30 Index & Stock & $70.5 \%$ & $69.1 \%$ & $85.2 \%$ & $99.2 \%$ & $98.5 \%$ & $65.3 \%$ \\
\hline EDP & Energia de Portugal & Stock & $75.9 \%$ & $69.0 \%$ & $88.0 \%$ & $94.9 \%$ & $86.8 \%$ & $52.4 \%$ \\
\hline EUR & Euro & Liquid Currency & $71.4 \%$ & $69.3 \%$ & $84.5 \%$ & $86.2 \%$ & $85.1 \%$ & $66.9 \%$ \\
\hline GALP & Galp Energia SGPS & Stock & $69.7 \%$ & $67.3 \%$ & $88.4 \%$ & $85.4 \%$ & $81.6 \%$ & $66.6 \%$ \\
\hline PLZ & Polish Zloty & Less Liq.. Currency & $87.6 \%$ & $86.6 \%$ & $85.7 \%$ & $99.7 \%$ & $99.2 \%$ & $83.3 \%$ \\
\hline CZK & Czech Koruna & Less Liq.. Currency & $88.3 \%$ & $87.1 \%$ & $86.6 \%$ & $99.8 \%$ & $99.5 \%$ & $92.6 \%$ \\
\hline \multirow[t]{5}{*}{ HUF } & Hungarian Fiorine & Less Liq.. Currency & $89.1 \%$ & $88.0 \%$ & $85.3 \%$ & $99.8 \%$ & $99.5 \%$ & $92.8 \%$ \\
\hline & Averages & Commodity & $68.5 \%$ & $65.7 \%$ & $87.4 \%$ & $91.1 \%$ & $90.6 \%$ & $71.9 \%$ \\
\hline & & Liquid Currency & $71.5 \%$ & $69.7 \%$ & $86.2 \%$ & $87.9 \%$ & $87.2 \%$ & $73.3 \%$ \\
\hline & & Stock & $69.8 \%$ & $67.1 \%$ & $87.2 \%$ & $95.7 \%$ & $92.6 \%$ & $65.3 \%$ \\
\hline & & Less Liq.. Currency & $82.4 \%$ & $80.5 \%$ & $86.6 \%$ & $96.7 \%$ & $94.1 \%$ & $90.6 \%$ \\
\hline
\end{tabular}

\section{Conclusion}

Any bank intending to calculate the own-funds requirements under the new IMA market risk framework, yet exposed to Bitcoin or any other cryptocurrency exposure, is faced with the hard task of answering the basic question of which risk factor category should the cryptocurrency risk factors be mapped. The aim of this work was to exactly that: to classify Bitcoin into an appropriate risk-class, with the purpose of determining market risk own-funds requirements. To this end, we examined price microstructure of the USD per Bitcoin, and compared it to other financial variables, as a proxy toward Bitcoin classification into the appropriate risk-class. Besides, the price discovery mechanism which is directly linked to the efficient market hypothesis has direct implications in the way markets, banks and supervisory authorities approach the issue of setting capital constraints in cryptocurrency operations.

To answer these open research questions of immense significance, we utilized a large dataset of high-frequency data. We analyzed these available datasets with the use of advanced machine learning techniques. Specifically, we employed venerable XGBOOST algorithms for the purpose of analyzing market liquidity and volatility patterns, and bleeding-edge deep learning techniques to scrutinize price discovery. 
Our main findings disprove the market efficiency hypothesis. In addition, from the market liquidity perspective, our findings show that Bitcoin behavior mostly resembles less liquid currencies. Concerning the detected volatility patterns, we showed that these do not substantially differ from other assets on a microstructure level.

These findings have serious regulatory implications. Indeed, under the revised standardized approach, the required liquidity horizons range from 10 to 120 days, depending on the classification of risk factor. This is in contrast to the global 10-day horizon, which is used without exception under the now-obsolete Internal Model Approach (VaR) framework. Under this regard, our results point that the 20-day liquidity horizon used for less liquid currencies may be more appropriate for Bitcoin rather than the 10-day liquidity horizon that is currently considered for liquid currency pairs as well as equity large caps. Therefore, our work points to a course of action that regulatory institutions must take. We emphasize, however, that further scrutiny is needed, by expanding our analysis to include more risk classes and more statistical metrics, before it is safe to adopt conclusive actions and policy recommendations. This remains to be addressed in our future research.

\section{References}

Balcilar, Mehmet., Elie, Bouri., Rangan, Gupta., and David, Roubaud (2017). Can volume predict Bitcoin returns and volatility? A quantiles-based approach. Economic Modelling. 64, 74-81.

Bariviera, Aurelio F (2017). The inefficiency of Bitcoin revisited: A dynamic approach. Economics Letters. 161, 1-4.

Cheah, Eng-Tuck., and John, Fry (2015). Speculative bubbles in Bitcoin markets? An empirical investigation into the fundamental value of Bitcoin. Economics Letters. 130, 32-36.

Cheah, Eng-Tuck., Mishra, Tapas., Parhi, Mamata., and Zhang, Zhuang (2018). Long memory interdependency and inefficiency in Bitcoin markets. Economics Letters.

Chen, Tianqi., and Guestrin, Carlos (2016). XGBoost: A Scalable Tree Boosting System. Proc. ACM KDD.

Cho, Kyunghyun., Merrienboer, Bart van., Gulcehre, Caglar., Bahdanau, Dzmitry., Bougares, Fethi., Schwenk, Holger., and Bengio, Yoshua (2014). Learning Phrase Representations using RNN Encoder-Decoder for Statistical Machine Translation. Proc. EMNLP.

Demir, Ender., Gozgor, Giray., Lau, Chi Keung, Marco., and Samuel, A.Vigne (2018). Does economic policy uncertainty predict the Bitcoin returns? An empirical investigation. Finance Research Letters.

Katsiampa, Paraskevi (2017). Volatility estimation for Bitcoin: A comparison of GARCH models. Economics Letters. 158, 3-6

Kingma, Diederik., Salimans, Tim., and Welling, Max (2015). Variational dropout and the local reparameterization trick. Proc. NIPS.

Lahmiri, Salim., Stelios, Bekiros., and Antonio, Salvi (2018). Long-range memory, distributional variation and randomness of Bitcoin volatility. Chaos, Solitons \& Fractals. 107, 43-48.

MacDonell, Alec (2014). Popping the Bitcoin bubble: An application of log-periodic power law modeling to digital currency. University of Notre Dame working paper.

Mosbeh, Hsini., and Mohamed, Nidhal MOSBAHI (2016). Stock market liquidity measurement via the Bid-ask spread: Tunis stockmarket. Business and Economic Research, Macrothink Institute, 6(2), 65-78.

Vinod, Nair., and Hinton, Geoffrey (2010). Rectified linear units improve restricted boltzmann machines. Proc. ICML.

Phillip, Andrew., Jennifer, Chan., and Shelton, Peiris (2018). A new look at Cryptocurrencies. Economics Letters. 163 , 6-9.

Pieters, Gina., and Sofia, Vivanco (2017). Financial regulations and price inconsistencies across Bitcoin markets. Information Economics and Policy. 39, 1-14.

Quadrana, Massimo., Karatzoglou, Alexandros., Hidasi, Balázs., and Cremonesi, Paolo (2017). Personalizing Sessionbased Recommendations with Hierarchical Recurrent Neural Networks. Proc. ASM RecSys.

See, Abigail., Liu, Peter J., and Manning, Christopher, D. (2017). Get to the point: Summarization with pointer-generator networks. Proc. ACL.

Tu, Zhaopeng., Lu, Zhengdong., Liu, Yang., Liu, Xiaohua., and Li, Hang (2016). Modeling coverage for neural machine translation. Proc. ACL.

Urquhart, Andrew (2017). Price clustering in Bitcoin. Economics Letters. 159, 145-148.

Urquhart, Andrew (2016). The inefficiency of Bitcoin. Economics Letters. 148, 80-82.

${ }^{1}$ https://www.dukascopy.com/swiss/english/marketwatch/historical/

Cite this article as: Vasilis Siakoulis, A nastasios Petropoulosand Panagiotis Lazaris(2021). Bitcoin economic behavior analysis and policy implications by leveraging deep learning and high-frequency data. International Journal of Data Science and Big Data Analytics. 1(1), 55-62. doi: 10.51483/IJDSBDA.1.1.2021.55-62. 